\title{
Latest Progresses in Allergic Diseases Biomarkers: Asthma and Atopic Dermatitis
}

\author{
Pier Giorgio Puzzovio and Francesca Levi-Schaffer* \\ Pharmacology and Experimental Therapeutics Unit, Faculty of Medicine, School of Pharmacy, Institute for Drug Research, The \\ Hebrew University of Jerusalem, Jerusalem, Israel
}

In the last years, the understanding of the pathologic mechanisms of asthma and atopic dermatitis, both characterized by allergic inflammation, has greatly improved. However, it is evident that both diseases present with high heterogeneity, which complicates the diagnosis and the therapeutic approach of the patients. Moreover, some of the currently available strategies to treat asthma and atopic dermatitis are still mostly controlling the symptoms, but not to lead towards full healing, thus having these two diseases labelled as unmet clinical needs by WHO. Therefore, the "one-size-fits-all" strategy is outdated for asthma and atopic dermatitis, and there is the need of better methods to clearly diagnose the disease and tailor the therapy according to the specific

OPEN ACCESS

Edited by: Ilaria Puxeddu, University of Pisa, Italy

Reviewed by:

Diana Boraschi,

Shenzhen Institutes of Advanced Technology (CAS), China

Paola Italiani,

National Research Council (CNR), Italy

*Correspondence: Francesca Levi-Schaffer francescal@ekmd.huji.ac.il

Specialty section: This article was submitted to Inflammation Pharmacology, a section of the journal Frontiers in Pharmacology

Received: 26 July 2021 Accepted: 20 September 2021 Published: 30 September 2021

Citation: Puzzovio PG and Levi-Schaffer $F$ (2021) Latest Progresses in Allergic Diseases Biomarkers: Asthma and Atopic Dermatitis.

Front. Pharmacol. 12:747364. doi: 10.3389/fphar.2021.747364 symptomatology. In this regard, the use of biomarkers has been advanced in order to characterize both diseases according to their clinical signs and to facilitate the subsequent treatment. Despite the advancements made in this regard, there is still need for better and more sensitive biomarkers and for less invasive sampling methodologies, with the aim to diagnose specifically each manifestation of asthma and atopic dermatitis and to provide the best treatment with the least suffering for the patients.

Keywords: allergic inflammation, asthma, atopic dermatitis, biomarkers, eosinophils, neutrophils

\section{INTRODUCTION}

In recent years, advancements of medical research in the field of allergic diseases have led to a better understanding that pathologies characterized by allergic inflammation (AI) are heterogeneous and present with a high degree of variability among patients (Roth and Stolz, 2019; Bakker et al., 2020). Moreover, most of the currently available treatments are still only able to ease the symptomatology/ symptoms, to decrease inflammation and some of them to partially prevent exacerbations and perhaps to modify the natural course of the disease. However, even the newest biologic-based drugs are not able to cure it. This might be due to the concomitant presence of an atopic condition together with the inability of the atopic individual to fully resolve the inflammation. Thus, allergic diseases and especially severe asthma and atopic dermatitis have been labelled by the WHO as unmet clinical needs (Breiteneder et al., 2019). Given the advances in research, and in light of the concept of personalized medicine, the necessity of finding novel and more accurate biomarkers for allergic diseases has been raised. A biomarker (or biological marker) is defined as a "characteristic that is objectively measured and evaluated as an indicator of normal biologic processes, pathogenic processes, or pharmacologic responses to a therapeutic intervention" according to the National Institute of Health. Consequently, biomarkers evaluation should help in the diagnosis of the disease as well as in predicting its outcomes and the effects of the prescribed therapy (Narendra et al., 2019). 


\section{BIOMARKERS FOR ASTHMA}

Asthma is a lung disease characterized by sometimes irreversible bronchoconstriction, airway hyperresponsiveness, chronic inflammation, mucus hypersecretion and tissue remodeling (Lambrecht and Hammad, 2015). In the last 40 years its prevalence and morbidity have increased, with approximately 300 million individuals affected worldwide and a total of $\$ 80$ billion dollars yearly expenses (Narendra et al., 2019). Over the years, several attempts have been made to better characterize the etiopathology of the disease, but still there is no effective therapy for all the spectrum of asthma forms, especially for the severe ones. Indeed in mild/moderate cases, asthma symptoms and underlying inflammation can mostly be controlled with the use of inhaled $\beta$-adrenergic agonists, muscarinic antagonists and glucocorticosteroids and other available anti-inflammatory drugs (Lambrecht and Hammad, 2015; Koarai and Ichinose, 2018). More recently, the use of monoclonal antibodies such as anti-IgE, anti-IL-5, anti-IL-5R, and anti-IL-4/IL-13R $\alpha$ has been demonstrated to be able to control asthma pathogenesis and hence symptoms, but not to resolve the disease. Thus, as mentioned above, asthma remains an unmet clinical need.

\section{Asthma Endotypes and Phenotypes}

In recent years, asthma has been defined as a disease characterized by heterogeneous features, which include the type of inflammation, presentation of the symptoms, response to treatments and long-term consequences for the patients. Therefore, asthma is better characterized by defining endotypes and phenotypes (Narendra et al., 2019; Roth and Stolz, 2019). Asthma endotypes encompass the pathologic mechanisms underlying the disease, while phenotypes include its clinical manifestations (Kuruvilla et al., 2019). Endotypes classification allows asthma to be divided into type 2 and nontype 2 asthma, although there is evidence of different subtypes linked to the inflammasome and skin structural components, and mixed 2, 1, 17 subtypes (Agache and Akdis, 2019). Asthma phenotypes include patients' features (age, gender, ethnicity, etc.), morphophysiological characteristics of the airways, response to therapies and clinical outcomes (Agache and Akdis, 2019). The emergence of the concept of asthma endotypes and phenotypes has prompted the need of a better understanding of the disease characteristics, in order to have a more personalized therapeutic approach and to predict the outcomes of the treatment. Therefore, the use of biomarkers to define the specific presentations of asthma has been advanced, each of them with their pros and cons.

\section{Biomarkers for Asthma}

In order to be eligible for asthma, a biomarker should be "superior, actionable, valuable, economical, and clinically deployable" (Diamant et al., 2019). Biomarkers for asthma are mainly divided into biomarkers for type 2 and non-type 2 asthma and might be sampled from different sources, with several advantages and disadvantages (Diamant et al., 2019; Narendra et al., 2019).

\section{Biomarkers for Type 2 Asthma Eosinophils}

One of the main biomarkers for type 2 asthma is the eosinophil (Eos) numbers, which are preferably analyzed in the blood and in the sputum of asthmatic patients due to the lower invasiveness of these methods, although with lower reproducibility and higher technical complexity (Diamant et al., 2019). Increased Eos counts in the blood $(>400$ cells $/ \mu l)$ were associated with higher prevalence of exacerbations and lower possibilities to control the disease (D. B. Price et al., 2015). However, Eos blood counts for asthma are not fully reliable, since blood eosinophilia might be due to other T2 inflammation-inducing conditions, such as parasitic infections or some autoimmune diseases (Narendra et al., 2019). Nevertheless, blood eosinophilia is a good marker to follow the T2 inflammation after treatment with anti-IL-5 (mepolizumab and reslizumab), anti-IL-5R $\alpha$ (benralizumab) and anti-IL-4 (dupilumab) biologics (Castro et al., 2011; Wenzel et al., 2013; Ortega et al., 2014; FitzGerald et al., 2016), as high blood Eos count is considered a good predictive value for the response to the aforementioned biologics (Cevhertas et al., 2020). Sputum Eos is believed to be the most accurate method to assess eosinophilic asthma (a value higher than $2 \%$ Eos is considered indicative of airway inflammation) (Westerhof et al., 2015; Walsh et al., 2016). A more refined technique to distinguish between type 2 and non-type 2 asthma involves analysis of the sputum mRNA levels of Th2 cytokines (Seys et al., 2017). Sputum Eos have been historically employed to follow the outcomes of corticosteroid treatment, since lower eosinophilia correlated with reduced exacerbations and hospitalizations after inhaled corticosteroids (ICS) administration (Morrow Brown, 1958; Green et al., 2002; Fitzpatrick et al., 2016). Another technique employed to analyze Eos in asthmatic patients is bronchoscopy, which is performed via biopsies, bronchoalveolar lavage or bronchial brushing. However, the higher invasiveness and complexity of these techniques limits their application (Diamant et al., 2019). Another possibility involves measurement of Eos granule proteins, such as Eos peroxidase, Eos cationic protein and Eos-derived neurotoxins, which have been found to decrease after administration of anti-Eos biologics (Narendra et al., 2019).

\section{Fraction of Exhaled Nitric Oxide}

FeNO measurement is an indirect indication of airway inflammation, since it correlates with Eos counts in the lungs (Fajt and Wenzel, 2015). Indeed, FeNO is linked to eosinophilia, according to the American Thoracic Society recommendations, $(>50 \mathrm{ppb}$ and $>30 \mathrm{ppb}$ for adults and children, respectively) (Dweik et al., 2011). FeNO is directly measurable in the exhaled breath of patients. The sampling method is easy and non-invasive, and the results are reproducible, prompting its use also for pediatric asthma (Neerincx et al., 2017). However, the results might be influenced by several factors, such as age, smoking habits, drug use, which should be taken into consideration when performing the measurement (Buchvald et al., 2005; Borrill et al., 2006). Even though it was found that FeNO levels decrease in response to inhaled corticosteroids (ICS) 
and dupilumab treatment (D. Price et al., 2013; Wenzel et al., 2013), the ERS/ATS guidelines suggest to avoid the use of FeNO as a predictive marker for therapy in severe asthma (Chung et al., 2014).

\section{Serum IgE}

Total serum IgE were found to be increased in allergic asthmatic adults and children, and their levels increased with disease severity (Burrows et al., 1995). Moreover, high serum IgE levels are indicative of sensitization to an allergen, which makes patients eligible for treatment with the monoclonal anti-IgE antibody omalizumab. However, it was demonstrated that, despite reducing serum $\operatorname{IgE}$, total $\operatorname{IgE}$ levels do not change significantly after omalizumab administration, due to the fact that omalizumab binds to free $\operatorname{IgE}$ and forms complexes with them, thus increasing the total IgE levels (Humbert et al., 2014). However, its use was found to reduce the incidence of asthma exacerbations (Humbert et al., 2014). Therefore, this marker does not allow to predict the response to treatments in asthmatic patients, and must be analyzed together with other biomarkers.

\section{Periostin}

Periostin is produced and released by epithelial cells after stimulation with IL-13, a cytokine indicative of Th2 inflammation (Izuhara et al., 2016). Periostin's effectiveness as a biomarker was shown to be higher than Eos, FeNO and serum IgE in patients with uncontrolled severe asthma and ICS treatment (Jia et al., 2012). Moreover, patients with high periostin levels were found to show reduced asthma exacerbations after treatment with lebrikizumab (anti-IL-13), demonstrating the prognostic value of serum periostin concentrations (Hanania et al., 2015). However, its levels fluctuate with age (Narendra et al., 2019) and can change also during other inflammatory processes such as atopic dermatitis, eosinophilic esophagitis and cancer, requiring its use in addition to other markers (Izuhara et al., 2016). In addition, the existence of different splicing variants of periostin complicates the measurement of this biomarker.

\section{Biomarkers for Non-type 2 Asthma Neutrophils}

Higher neutrophil counts in the sputum and in the blood have been associated with severe forms of asthma (Moore et al., 2014; Ricciardolo et al., 2018). However, there is no clear definition of neutrophilic asthma, since different threshold values for neutrophil levels were reported in the literature (Simpson et al., 2006; Moore et al., 2014). Moreover, airway neutrophilia was also found to be induced by use of oral corticosteroids (Alam et al., 2017), but also by other conditions, such as obesity, smoking habits, gastroesophageal reflux or lung infections (Ray and Kolls, 2017). This complicates the diagnosis of neutrophilic asthma, since some of its features are mistakenly ascribed to other diseases, such as chronic obstructive pulmonary disease, or effects of smoking (Gibson and Foster, 2019). Generally, a value between 61 and $76 \%$ is considered indicative of neutrophilic airway inflammation, although no real consensus exists about these threshold values (F. Schleich et al., 2016).

\section{Serum Cytokines}

The main markers associated with neutrophilic asthma are IL-17, IL-8, and $\mathrm{TNF} \alpha$, since their levels in the serum were found increased in asthmatic patients with neutrophilia (Diamant et al., 2019). A positive correlation was found between neutrophil numbers and IL-17 mRNA levels in the sputum (Bullens et al., 2006), and it was found that bronchial epithelial cells released IL-8 after stimulation with IL-17, contributing indirectly to IL-8-induced neutrophils recruitment (Lindén, 2001). However, this marker did not show any therapeutical predictive value since treatment with an anti-IL-17R biologic, brodalumab, did not prove to be effective in severe asthma (Busse et al., 2013). Tightly linked to airway neutrophilia is the cytokine IL-8, since it is known to be a chemoattractant for neutrophils and it has been found in high levels in BAL and sputum uncontrolled asthmatic patients (Gao et al., 2017). Moreover, expression of IL-8 receptors CXCR1-2 was found to be increased in the sputum of neutrophilic asthmatic patients (Wood et al., 2012). In this regard, the use of CXCR2 antagonists was shown to decrease neutrophils count in the sputum and to reduce mild exacerbations (Nair et al., 2012), further bolstering the connection between neutrophils and asthma. Other cytokines involved in neutrophilic asthma include IL-4, which might indirectly induce neutrophil migration and activation by regulating the expression levels of IL-8, TNF- $\alpha$, and IL- $1 \beta$ in correlation with the severity of the disease (LavoieLamoureux et al., 2010). Therefore, IL- 8 and IL-4 might be helpful in distinguishing between airway neutrophilia due to asthma or other pathological conditions. TNFa levels were found to be increased in the sputum of neutrophilic asthmatics (Simpson et al., 2007) and to positively correlate with both $\mathrm{NO}$ and neutrophil numbers in severe asthmatic patients (Silvestri et al., 2006). Interestingly, treatment with the anti-TNFa etanercept improves airway hyperresponsiveness and quality of life in refractory asthma, and this improvement positively correlated with etanerceptinduced reduction of membrane-bound TNFa expression (Brightling et al., 2008). Thus, TNFa might be used also as a predictive biomarker for etanercept therapy. Other markers are related to neutrophil activation and include sputum myeloperoxidase and elastase, which were detected in high levels in neutrophilic asthma (F. Schleich et al., 2016).

\section{Novel Biomarkers}

\section{Airway Remodeling}

One of the main features of asthma is the airway remodeling, involving airways obstruction, mucus hypersecretion, angiogenesis, and excessive fibrosis (Bergeron et al., 2009). Unfortunately, at the moment there is no clear-cut marker for airway remodeling, and the preferred method to analyze remodeling in the airways is the bronchial biopsy, which is highly invasive and risky. Furthermore, the great variability in the tissue raises the necessity of having more than one sample (Diamant et al., 2019). Some less-invasive markers include 
sputum matrix metalloproteinase 2 (MMP-2), fibroblast growth factor 2 (FGF-2) and galectin-3, the latter being predictive of omalizumab effects on airway remodeling (Mauri et al., 2014; Elkolaly and Ali, 2018; Sivakoti et al., 2018; Tan et al., 2020). Other airways remodeling markers include CCL16, released by bronchiolar exocrine cells, which is usually measured in the sputum and compared to IL-8 levels (F. Schleich et al., 2016).

\section{Volatile Organic Compounds}

VOCs are a collection of molecules derived from the metabolism of different endogenous or exogenous compounds. These molecules were found to differ between eosinophilic and neutrophilic asthma. For example, hexane and 2-hexanone were found to be characteristic of eosinophilic asthma, with similar accuracy to FeNO and blood Eos (F. N. Schleich et al., 2019). On the other hand, the molecules found in high concentrations in neutrophilic asthma are nonanal, 1-propanol and hexane (F. N. Schleich et al., 2019).

\section{Specialized Pro-resolving Mediators}

SPMs are a class of lipid molecules encompassing different families with their biosynthetic pathways and receptors, all of them implicated in the resolution of inflammation (Fullerton and Gilroy, 2016). SPMs levels might be analyzed in a wide number of biological materials, such as blood, sputum, bronchoalveolar lavage (BAL), exhaled breath condensates, as well as in urine, breast milk and tears, in their bioactive concentration $(\mathrm{pg} / \mathrm{ml})$ (Serhan, 2014). Notably, SPMs pathways are reduced in severe asthma patients. Specifically, lower lipoxin A4 (LXA4) levels were found in the BAL of severe asthmatic patients (Planaguma et al., 2008), correlating with decreased lung functions. Moreover, severe asthma patients have been found to present with reduced docosahexaenoic acid (DHA) concentrations in the airways' mucosa, hinting that production of protectin D1 and D-resolvins might be impaired as well (Freedman et al., 2004). It was also found that in severe asthma the expression of ALX/ FPR2, the receptor binding resolvin D1 and LXA4, is reduced on peripheral blood Eos and neutrophils and increased on BAL macrophages and neutrophils and peripheral blood natural killer (NK) cells (Planaguma et al., 2008; Barnig et al., 2013; Ricklefs et al., 2017). This evidence would indicate that SPMs and their pathways might be a good candidate for detecting severe asthma and the consequent defective resolution.

\section{A Potential New Marker: $s C D 48$}

CD48 is an activating receptor expressed on immune cells which exists in a membrane-bound form and a soluble one (sCD48) (Smith et al., 1997). CD48 on mast cells was found to interact with CD244 on Eos, initiating a cross-talk with marked proinflammatory outcomes in AI, the Allergic Effector Unit (Elishmereni et al., 2013). The expression of CD48 was found to be increased in Eos from peripheral blood and nasal polyps of mild asthmatic patients (Munitz et al., 2006a) and on NK cells, B-cells and T-cells of severe asthmatic patients (Gangwar et al., 2017). The levels of CD48 soluble form, sCD48, were higher in the serum of mild asthmatic patients and reduced in moderate and severe asthma (Gangwar et al., 2017). Interestingly, sCD48 levels in asthmatic patients did not correlate with Th2 inflammation markers, and this prompted the hypothesis that its expression might be linked to a broader role in inflammatory processes rather than specific AI (Breuer et al., 2018). Therefore, CD48 might be a good candidate as a biomarker for different degrees of asthma severity.

\section{BIOMARKERS FOR ATOPIC DERMATITIS}

Atopic dermatitis $(\mathrm{AD})$ is among the most common inflammatory skin diseases (Nomura et al., 2020). The lack of a proper therapeutic strategy against $\mathrm{AD}$ has rendered this disease a significant socioeconomic burden worldwide, with higher prevalence amongst children (Barbarot et al., 2018). In AD there is increasing evidence pointing to a high degree of heterogeneity in clinical manifestations and molecular characteristics, advancing the concepts of endotypes/subtypes also for AD (Bakker et al., 2020). As with asthma, treatments for $\mathrm{AD}$ are moving towards the concept of personalized medicine, mostly due to the heterogeneity of the disease. This is most important, since AD is still dealt with the "one-size-fits-all" approach, which greatly limits the effectiveness of the treatment (Bieber et al., 2017).

\section{AD Subtypes}

Over the years, the characterization of $\mathrm{AD}$ has been significantly elucidated thereby shedding light on the complexity of this disease. This led to the classification of $\mathrm{AD}$ manifestations into different subtypes, namely age-related features, severity of the disease, age of onset and ethnicity according to skin condition, presence of lesions, and underlying inflammation (Bieber et al., 2017).

This classification is mainly based on the severity and the extension of the lesions and of the skin conditions, and it employs diagnostic scores such as the SCORAD or Eczema Area and Severity Index (Bieber et al., 2017). However, the underlying inflammatory response in the patients is also taken into consideration and used to define the disease characteristics. The immunological profile of $\mathrm{AD}$ patients shows a marked Th2 inflammation in all the subtypes, as shown by frequencies of $\mathrm{IL}-13^{+}$and IL- $4^{+}$T-cells (Esaki et al., 2016b), while Th22 inflammation increased from infancy to adulthood, as shown by high levels of IL-22 in adult $\mathrm{AD}$ in comparison to infancy AD (Czarnowicki et al., 2020). In childhood AD, Th17 and Th9 responses were found, as demonstrated by the higher levels of cytokines such as IL17A, IL-19 and IL-9, respectively (Esaki et al., 2016a). This immunological response might change according to the ethnicity of the patients. Asian patients present with increased Th17/Th22 inflammation, shown by the increased skin thickness and Th17/22 markers expression in skin and blood, with "psoriasis-like" manifestations (Noda et al., 2015). On the other hand, African Americans displayed increased Th22 response and skin barrier defects, while Caucasian patients showed induction of Th22, Th17 and Th1 inflammation, with reduced production of skin barrier 
proteins (Nomura et al., 2020). In all ethnicities the Th2 response was always present.

This evidence shows that $\mathrm{AD}$ heterogeneity comprises many factors, that complicate the diagnosis and the consequent treatment. Therefore, as for asthma, also in $\mathrm{AD}$ biomarkers have been proposed to facilitate the definition of the disease severity.

\section{AD Biomarkers}

In contrast to what was seen in asthma, there is a general lack of suitable biomarkers for $\mathrm{AD}$, mostly due to the difficulties inherent to sample retrieval. Indeed, most of the existing knowledge regarding $\mathrm{AD}$ biomarkers is obtained from studies performed on skin biopsies, which is an invasive and potentially dangerous method especially for infants. Therefore, new sampling methods are being used, such as skin tape-stripping, for following both the disease and the treatments (Castelo-Soccio, 2019; GuttmanYassky et al., 2019). Another source of samples for AD biomarkers analysis is the serum of the patients (Ungar et al., 2017). Other sampling methods, less invasive, are dried blood spots (DBS), consisting in droplets of blood collected via a capillary and absorbed on a cellulose layer. DBS are then eluted via an adequate buffer and processed for biomarkers analysis. This technique is minimally painful and easy to process (J. L. Thijs et al., 2019). Another less invasive source of samples is saliva, mainly due to the possibility of blood biomarkers diffusing into the salivary glands (Thijs J. et al., 2015). Biomarkers for $\mathrm{AD}$ in the skin are generally measured via their mRNA levels, while serum biomarkers can be analyzed also via ELISA (Ungar et al., 2017; Guttman-Yassky et al., 2019). Identification of potential patients before symptoms appearance involves evaluation of the skin barrier functionality, via epidermal water loss and expression of FLG1-2, encoding for the proteins filaggrin 1 and 2, or other structural skin proteins (Margolis et al., 2014; Bager et al., 2016). The stratification of patients is done also according to the underlying immunological response. AD is mainly a Th2driven disease, characterized by expression of IL-4, IL- 5 and IL-13, and the levels of these cytokines can also predict the outcomes of anti-IL-4, anti-IL-5 and anti-IL-13 biologics therapies (Bakker et al., 2020). Where the phenotype is more Th17-dominant, the levels of IL-17 are evaluated, and might be a good marker for following anti-IL-17-directed approaches (Bieber et al., 2017). In patients showing upregulation of the Th22 response, IL-22 is the most evaluated marker for disease severity, and its levels were also shown to be predictive of fezakinumab (anti-IL-22) administration outcomes (Brunner et al., 2019). Other markers for AD severity include ECP, TSLP, $\beta$-defensin 1, eotaxin, RANTES, CCL17-22-27, the latter allowing T-cells homing to the skin (Bieber et al., 2017; Bakker et al., 2020). Among these markers, CCL17 was the one showing the highest correlation with $\mathrm{AD}$ severity, although its levels are generally variable with $\mathrm{AD}$ heterogeneity and underlying inflammatory pathways (Landheer et al., 2014; Thijs J. L. et al., 2015). Sensitization to the allergen is measured via total and specific IgE, however the IgE profile is subject to great variation among the population. Therefore, it would be more useful to examine the ratio between specific and total IgE (Bieber et al., 2017).

\section{New Potential Biomarkers Adipokines}

In a recent study, the levels of serum adipokines were evaluated and related to the disease features. Two adipokines, adiponectin and resistin, showed lower levels in $\mathrm{AD}$ patients and an inverse proportionality trend with the severity of the disease. On the other hand, leptin levels were increased in AD patients, but did not correlate with disease severity. No correlation between adipokines' levels and patients' characteristics (age, gender, BMI) was found (Jaworek et al., 2020). Thus, although requiring more studies, adipokines might be a new interesting and more specific set of biomarkers linked to $\mathrm{AD}$ severity.

\section{CD300a}

$\mathrm{CD} 300 \mathrm{a}$ is an inhibitory receptor expressed on the surface of several immune cells, and its role in downregulating AI has been extensively demonstrated (Bachelet et al., 2005; Bachelet et al., 2006; Munitz et al., 2006b). It was recently published that total CD300a expression is increased in lesional AD skin and specifically on Eos, and that its expression positively correlated with hypoxic conditions and angiogenesis in AD skin (Karra et al., 2019). Moreover, CD300a expression was significantly increased on B-cells from $\mathrm{AD}$ patients and decreased on circulating NK cells (Karra et al., 2019). Interestingly, CD300a expression was not increased in non-lesional AD skin [(Karra et al., 2019) supporting information)], hinting that this receptor might be a marker for severe forms of the disease.

\section{CD48}

CD48 surface levels were found to be significantly decreased in peripheral blood from mild/moderate/severe $\mathrm{AD}$ patients, and on Eos, neutrophils, monocytes, basophils, NK cells, T- and B-cells (Minai-Fleminger et al., 2014). However, its expression was significantly increased on Eos in biopsies from lesional AD skin (Minai-Fleminger et al., 2014). It was hypothesized that this differential CD48 expression might be the result of CD48 sensitivity to local stimuli rather than systemic ones (MinaiFleminger et al., 2014). Thus, CD48 might provide information regarding local inflammation in $\mathrm{AD}$ lesional skin.

\section{Skin Microbiome}

The role of secondary infections in $\mathrm{AD}$, especially from bacteria such as Staphylococcus aureus, normally residing on the skin, is well characterized (Weidinger and Novak, 2016). A recent study has shown that $\mathrm{AD}$ skin presents with dysbiosis in comparison to healthy controls, with prevalence of Staphylococcus aureus and reduction in anaerobic bacteria species, correlating with disease severity (Fyhrquist et al., 2019). In another study, the skin microbiome composition was employed to divide patients in "dermotypes", each one with distinct bacterial genera prevalence and metabolic profiles (Tay et al., 2020). Among the dermotypes, the " $\mathrm{B}$ " one presented with higher Th2specific mediators, worsened symptomatology and increased possibility to develop other atopic diseases (Tay et al., 2020). 
This evidence adds another level of characterization of $\mathrm{AD}$, which might aid in stratification of the patients and evaluation of biomarkers.

\section{CONCLUDING REMARKS}

Since the emergence of the concept of personalized medicine, it has become clear that the "one-size-fits-all" approach for allergic diseases is not adequate to treat the high heterogeneity of patients. Thus, the search for biomarkers for predicting the occurrence and the outcomes of the disease was prompted. Despite the vast amount of research conducted, there is still a need for less invasive sampling techniques and more sensitive markers.

New techniques include the use of -omics technology, such as transcriptomics, proteomics and metabolomics, to create a detailed profile of the asthma features. One of the latest applications is the metabolic profiling of the breath of asthma patients before and after ICS treatment (Ferraro et al., 2020). Moreover, the aim of the ongoing SMART clinical trial (NCT04194814), started in 2019, is to test new non-invasive methods to evaluate biomarkers for skin structure and function variations.

In conclusion, biomarkers for asthma and $\mathrm{AD}$ provide useful tools in the diagnosis of the disease and the prediction of the

\section{REFERENCES}

Agache, I., and Akdis, C. A. (2019). Precision Medicine and Phenotypes, Endotypes, Genotypes, Regiotypes, and Theratypes of Allergic Diseases. J. Clin. Invest. 129 (4), 1493-1503. doi:10.1172/JCI124611

Alam, R., Good, J., Rollins, D., Verma, M., Chu, H., Pham, T. H., et al. (2017). Airway and Serum Biochemical Correlates of Refractory Neutrophilic Asthma. J. Allergy Clin. Immunol. 140 (4), 1004-e13. doi:10.1016/j.jaci.2016.12.963

Bachelet, I., Munitz, A., Moretta, A., Moretta, L., and Levi-Schaffer, F. (2005). The Inhibitory Receptor IRp60 (CD300a) Is Expressed and Functional on Human Mast Cells. J. Immunol. 175 (12), 7989-7995. Retrieved from: http://www.ncbi. nlm.nih.gov/pubmed/16339535. doi:10.4049/jimmunol.175.12.7989

Bachelet, I., Munitz, A., and Levi-Schaffer, F. (2006). Abrogation of Allergic Reactions by a Bispecific Antibody Fragment Linking IgE to CD300a. J. Allergy Clin. Immunol. 117 (6), 1314-1320. doi:10.1016/j.jaci.2006.04.031

Bager, P., Wohlfahrt, J., Boyd, H., Thyssen, J. P., and Melbye, M. (2016). The Role of Filaggrin Mutations during Pregnancy and Postpartum: Atopic Dermatitis and Genital Skin Diseases. Allergy 71 (5), 724-727. doi:10.1111/all.12849

Bakker, D. S., Nierkens, S., Knol, E. F., Giovannone, B., Delemarre, E. M., van der Schaft, J., et al. (2021). Confirmation of Multiple Endotypes in Atopic Dermatitis Based on Serum Biomarkers. J. Allergy Clin. Immunol. 147, 189-198. doi:10.1016/j.jaci.2020.04.062

Barbarot, S., Auziere, S., Gadkari, A., Girolomoni, G., Puig, L., Simpson, E. L., et al. (2018). Epidemiology of Atopic Dermatitis in Adults: Results from an International Survey. Allergy 73 (6), 1284-1293. doi:10.1111/all.13401

Barnig, C., Cernadas, M., Dutile, S., Liu, X., Perrella, M. A., Kazani, S., et al. (2013). Lipoxin A4 Regulates Natural Killer Cell and Type 2 Innate Lymphoid Cell Activation in Asthma. Sci. Transl Med. 5 (174), 174ra26. doi:10.1126/ scitranslmed.3004812

Bergeron, C., Al-Ramli, W., and Hamid, Q. (2009). Remodeling in Asthma. Proc. Am. Thorac. Soc. 6 (3), 301-305. doi:10.1513/pats.200808-089RM

Bieber, T., D’Erme, A. M., Akdis, C. A., Traidl-Hoffmann, C., Lauener, R., Schäppi, G., et al. (2017). Clinical Phenotypes and Endophenotypes of Atopic Dermatitis: Where Are We, and where Should We Go? J. Allergy Clin. Immunol. 139 (4), S58-S64. doi:10.1016/j.jaci.2017.01.008 symptoms' occurrence and therapeutical responses/outcomes. Novel methodologies for both sampling and analysis are now being evaluated. In time, this might result in better analytic strategies that would benefit both the patient and the clinician in terms of non-invasiveness, reliability and specificity of the marker, in order to design the best therapeutical approach for each patient.

\section{AUTHOR CONTRIBUTIONS}

PGP wrote the draft of the review; FL-S corrected it, and received grant funds.

\section{FUNDING}

FL-S receives funding for her research from the United StatesIsrael Binational Science Foundation (BSF) (Grant no. 2015045), Rosetrees Trust (United Kingdom), Aimwell Charitable Trust (United Kingdom), Israel Science Foundation (Grant no 442/18), Emalie Gutterman Memorial Endowed Fund (United States). FL-S is affiliated with the Adolph and Klara Brettler Center for Molecular Pharmacology and Therapeutics at the School of Pharmacy of The Hebrew University of Jerusalem.

Borrill, Z., Clough, D., Truman, N., Morris, J., Langley, S., and Singh, D. (2006). A Comparison of Exhaled Nitric Oxide Measurements Performed Using Three Different Analysers. Respir. Med. 100 (8), 1392-1396. doi:10.1016/j.rmed.2005.11.018

Breiteneder, H., Diamant, Z., Eiwegger, T., Fokkens, W. J., Traidl-Hoffmann, C., Nadeau, K., et al. (2019). Future Research Trends in Understanding the Mechanisms Underlying Allergic Diseases for Improved Patient Care. Allergy 74 (12), 2293-2311. doi:10.1111/all.13851

Breuer, O., Gangwar, R. S., Seaf, M., Barhoum, A., Kerem, E., and Levi-Schaffer, F. (2018). Evaluation of Soluble CD48 Levels in Patients with Allergic and Nonallergic Asthma in Relation to Markers of Type 2 and Non-type 2 Immunity: An Observational Study. J. Immunol. Res. 2018, 4236263-4236267. doi:10.1155/2018/4236263

Brightling, C., Berry, M., and Amrani, Y. (2008). Targeting TNF-Alpha: a Novel Therapeutic Approach for Asthma. J. Allergy Clin. Immunol. 121 (1), 5-2. doi:10.1016/j.jaci.2007.10.028

Brunner, P. M., Pavel, A. B., Khattri, S., Leonard, A., Malik, K., Rose, S., et al. (2019). Baseline IL-22 Expression in Patients with Atopic Dermatitis Stratifies Tissue Responses to Fezakinumab. J. Allergy Clin. Immunol. 143 (1), 142-154. doi:10.1016/j.jaci.2018.07.028

Buchvald, F., Baraldi, E., Carraro, S., Gaston, B., De Jongste, J., Pijnenburg, M. W., et al. (2005). Measurements of Exhaled Nitric Oxide in Healthy Subjects Age 4 to 17 Years. J. Allergy Clin. Immunol. 115 (6), 1130-1136. doi:10.1016/j.jaci.2005.03.020

Bullens, D. M., Truyen, E., Coteur, L., Dilissen, E., Hellings, P. W., Dupont, L. J., et al. (2006). IL-17 mRNA in Sputum of Asthmatic Patients: Linking T Cell Driven Inflammation and Granulocytic Influx? Respir. Res. 7 (1), 135. doi:10.1186/1465-9921-7-135

Burrows, B., Martinez, F. D., Cline, M. G., and Lebowitz, M. D. (1995). The Relationship between Parental and Children's Serum IgE and Asthma. Am. J. Respir. Crit. Care Med. 152 (5), 1497-1500. doi:10.1164/ajrccm.152.5.7582283

Busse, W. W., Holgate, S., Kerwin, E., Chon, Y., Feng, J., Lin, J., et al. (2013). Randomized, Double-Blind, Placebo-Controlled Study of Brodalumab, a Human anti-IL-17 Receptor Monoclonal Antibody, in Moderate to Severe Asthma. Am. J. Respir. Crit. Care Med. 188 (11), 1294-1302. doi:10.1164/rccm.201212-2318OC

Castelo-Soccio, L. (2019). Stripping Away Barriers to Find Relevant Skin Biomarkers for Pediatric Atopic Dermatitis. JAMA Dermatol. 155 (12), 1342. doi:10.1001/jamadermatol.2019.2792

Castro, M., Mathur, S., Hargreave, F., Boulet, L. P., Xie, F., Young, J., et al. (2011). Reslizumab for Poorly Controlled, Eosinophilic Asthma: a Randomized, 
Placebo-Controlled Study. Am. J. Respir. Crit. Care Med. 184 (10), 1125-1132. doi:10.1164/rccm.201103-0396OC

Cevhertas, L., Ogulur, I., Maurer, D. J., Burla, D., Ding, M., Jansen, K., et al. (2020). Advances and Recent Developments in Asthma in 2020. Allergy 75, 3124-3146. doi:10.1111/all.14607

Chung, K. F., Wenzel, S. E., Brozek, J. L., Bush, A., Castro, M., Sterk, P. J., et al. (2014). International ERS/ATS Guidelines on Definition, Evaluation and Treatment of Severe Asthma. Eur. Respir. J. 43 (2), 343-373. doi:10.1183/09031936.00202013

Czarnowicki, T., He, H., Canter, T., Han, J., Lefferdink, R., Erickson, T., et al. (2020). Evolution of Pathologic T-Cell Subsets in Patients with Atopic Dermatitis from Infancy to Adulthood. J. Allergy Clin. Immunol. 145 (1), 215-228. doi:10.1016/j.jaci.2019.09.031

Diamant, Z., Vijverberg, S., Alving, K., Bakirtas, A., Bjermer, L., Custovic, A., et al. (2019). Toward Clinically Applicable Biomarkers for Asthma: An EAACI Position Paper. Allergy 74 (10), 1835-1851. doi:10.1111/all.13806

Dweik, R. A., Boggs, P. B., Erzurum, S. C., Irvin, C. G., Leigh, M. W., Lundberg, J. O., et al. (2011). An Official ATS Clinical Practice Guideline: Interpretation of Exhaled Nitric Oxide Levels (FENO) for Clinical Applications. Am. J. Respir. Crit. Care Med. 184 (5), 602-615. doi:10.1164/rccm.9120-11ST

Elishmereni, M., Bachelet, I., Nissim Ben-Efraim, A. H., Mankuta, D., and LeviSchaffer, F. (2013). Interacting Mast Cells and Eosinophils Acquire an Enhanced Activation State In Vitro. Allergy 68 (2), 171-179. doi:10.1111/all.12059

Elkolaly, R., and Ali, D. (2018). "Galectn-3 : Marker of Airway Inflammation in Bronchial Asthma," in Airway Cell Biology and Immunopathology (Sheffield, England: European Respiratory Society), PA954. doi:10.1183/13993003.congress2018.PA954

Esaki, H., Brunner, P. M., Renert-Yuval, Y., Czarnowicki, T., Huynh, T., Tran, G., et al. (2016a). Early-onset Pediatric Atopic Dermatitis Is TH2 but Also TH17 Polarized in Skin. J. Allergy Clin. Immunol. 138 (6), 1639-1651. doi:10.1016/j.jaci.2016.07.013

Esaki, H., Czarnowicki, T., Gonzalez, J., Oliva, M., Talasila, S., Haugh, I., et al. (2016b). Accelerated T-Cell Activation and Differentiation of Polar Subsets Characterizes Early Atopic Dermatitis Development. J. Allergy Clin. Immunol. 138 (5), 1473-e5. doi:10.1016/j.jaci.2016.04.052

Fajt, M. L., and Wenzel, S. E. (2015). Asthma Phenotypes and the Use of Biologic Medications in Asthma and Allergic Disease: The Next Steps toward Personalized Care. J. Allergy Clin. Immunol. 135 (2), 299-311. doi:10.1016/j.jaci.2014.12.1871

Ferraro, V. A., Carraro, S., Pirillo, P., Gucciardi, A., Poloniato, G., Stocchero, M., et al. (2020). Breathomics in Asthmatic Children Treated with Inhaled Corticosteroids. Metabolites 10 (10), 390. doi:10.3390/metabo10100390

FitzGerald, J. M., Bleecker, E. R., Nair, P., Korn, S., Ohta, K., Lommatzsch, M., et al. (2016). Benralizumab, an Anti-interleukin-5 Receptor a Monoclonal Antibody, as Add-On Treatment for Patients with Severe, Uncontrolled, Eosinophilic Asthma (CALIMA): a Randomised, Double-Blind, Placebo-Controlled Phase 3 Trial. Lancet 388 (10056), 2128-2141. doi:10.1016/S0140-6736(16)31322-8

Fitzpatrick, A. M., Jackson, D. J., Mauger, D. T., Boehmer, S. J., Phipatanakul, W., Sheehan, W. J., et al. (2016). Individualized Therapy for Persistent Asthma in Young Children. J. Allergy Clin. Immunol. 138 (6), 1608-e12. doi:10.1016/ j.jaci.2016.09.028

Freedman, S. D., Blanco, P. G., Zaman, M. M., Shea, J. C., Ollero, M., Hopper, I. K., et al. (2004). Association of Cystic Fibrosis with Abnormalities in Fatty Acid Metabolism. N. Engl. J. Med. 350 (6), 560-569. doi:10.1056/NEJMoa021218

Fullerton, J. N., and Gilroy, D. W. (2016). Resolution of Inflammation: a New Therapeutic Frontier. Nat. Rev. Drug Discov. 15 (8), 551-567. doi:10.1038/nrd.2016.39

Fyhrquist, N., Muirhead, G., Prast-Nielsen, S., Jeanmougin, M., Olah, P., Skoog, T., et al. (2019). Microbe-host Interplay in Atopic Dermatitis and Psoriasis. Nat. Commun. 10 (1), 4703. doi:10.1038/s41467-019-12253-y

Gangwar, R. S., Minai-Fleminger, Y., Seaf, M., Gutgold, A., Shikotra, A., Barber, C., et al. (2017). CD48 on Blood Leukocytes and in Serum of Asthma Patients Varies with Severity. Allergy 72 (6), 888-895. doi:10.1111/all.13082

Gao, H., Ying, S., and Dai, Y. (2017). Pathological Roles of Neutrophil-Mediated Inflammation in Asthma and its Potential for Therapy as a Target. J. Immunol. Res. 2017, 1-12. doi:10.1155/2017/3743048

Gibson, P. G., and Foster, P. S. (2019). Neutrophilic Asthma: welcome Back!. Eur. Respir. J. 54 (5), 1901846. doi:10.1183/13993003.01846-2019

Green, R. H., Brightling, C. E., McKenna, S., Hargadon, B., Parker, D., Bradding, P., et al. (2002). Asthma Exacerbations and Sputum Eosinophil Counts: a Randomised Controlled Trial. Lancet 360 (9347), 1715-1721. doi:10.1016/ S0140-6736(02)11679-5
Guttman-Yassky, E., Diaz, A., Pavel, A. B., Fernandes, M., Lefferdink, R., Erickson, T., et al. (2019). Use of Tape Strips to Detect Immune and Barrier Abnormalities in the Skin of Children with Early-Onset Atopic Dermatitis. JAMA Dermatol. 155 (12), 1358. doi:10.1001/jamadermatol.2019.2983

Hanania, N. A., Noonan, M., Corren, J., Korenblat, P., Zheng, Y., Fischer, S. K., et al. (2015). Lebrikizumab in Moderate-To-Severe Asthma: Pooled Data from Two Randomised Placebo-Controlled Studies. Thorax 70 (8), 748-756. doi:10.1136/thoraxjnl-2014-206719

Humbert, M., Busse, W., Hanania, N. A., Lowe, P. J., Canvin, J., Erpenbeck, V. J., et al. (2014). Omalizumab in Asthma: An Update on Recent Developments. J. Allergy Clin. Immunol. Pract. 2 (5), 525-36.e1. doi:10.1016/j.jaip.2014.03.010

Izuhara, K., Conway, S. J., Moore, B. B., Matsumoto, H., Holweg, C. T., Matthews, J. G., et al. (2016). Roles of Periostin in Respiratory Disorders. Am. J. Respir. Crit. Care Med. 193 (9), 949-956. doi:10.1164/rccm.201510-2032PP

Jaworek, A. K., Szepietowski, J. C., Szafraniec, K., Jaworek, M., Hałubiec, P., WojasPelc, A., et al. (2020). Adipokines as Biomarkers of Atopic Dermatitis in Adults. J. Clin. Med. 9 (9), 2858. doi:10.3390/jcm9092858

Jia, G., Erickson, R. W., Choy, D. F., Mosesova, S., Wu, L. C., Solberg, O. D., et al. (2012). Periostin Is a Systemic Biomarker of Eosinophilic Airway Inflammation in Asthmatic Patients. J. Allergy Clin. Immunol. 130 (3), 647-e10. doi:10.1016/j.jaci.2012.06.025

Karra, L., Gangwar, R. S., Puzzovio, P. G., Fyhrquist, N., Minai-Fleminger, Y., Landolina, N., et al. (2019). CD300a Expression Is Modulated in Atopic Dermatitis and Could Influence the Inflammatory Response. Allergy 74 (7), 1377-1380. doi:10.1111/all.13724

Koarai, A., and Ichinose, M. (2018). Possible Involvement of AcetylcholineMediated Inflammation in Airway Diseases. Allergol. Int. 67 (4), 460-466. doi:10.1016/j.alit.2018.02.008

Kuruvilla, M. E., Lee, F. E., and Lee, G. B. (2019). Understanding Asthma Phenotypes, Endotypes, and Mechanisms of Disease. Clin. Rev. Allergy Immunol. 56 (2), 219-233. doi:10.1007/s12016-018-8712-1

Lambrecht, B. N., and Hammad, H. (2015). The Immunology of Asthma. Nat. Immunol. 16 (1), 45-56. doi:10.1038/ni.3049

Landheer, J., de Bruin-Weller, M., Boonacker, C., Hijnen, D., Bruijnzeel-Koomen, C., and Röckmann, H. (2014). Utility of Serum Thymus and Activation-Regulated Chemokine as a Biomarker for Monitoring of Atopic Dermatitis Severity. J. Am. Acad. Dermatol. 71 (6), 1160-1166. doi:10.1016/j.jaad.2014.07.031

Lavoie-Lamoureux, A., Moran, K., Beauchamp, G., Mauel, S., Steinbach, F., Lefebvre-Lavoie, J., et al. (2010). IL-4 Activates Equine Neutrophils and Induces a Mixed Inflammatory Cytokine Expression Profile with Enhanced Neutrophil Chemotactic Mediator Release Ex Vivo. Am. J. Physiol. Lung Cel Mol Physiol 299 (4), L472-L482. doi:10.1152/ajplung.00135.2009

Lindén, A. (2001). Role of Interleukin-17 and the Neutrophil in Asthma. Int. Arch. Allergy Immunol. 126 (3), 179-184. doi:10.1159/000049511

Margolis, D. J., Gupta, J., Apter, A. J., Ganguly, T., Hoffstad, O., Papadopoulos, M., et al. (2014). Filaggrin-2 Variation Is Associated with More Persistent Atopic Dermatitis in African American Subjects. J. Allergy Clin. Immunol. 133 (3), 784-789. doi:10.1016/j.jaci.2013.09.015

Mauri, P., Riccio, A. M., Rossi, R., Di Silvestre, D., Benazzi, L., De Ferrari, L., et al. (2014). Proteomics of Bronchial Biopsies: Galectin-3 as a Predictive Biomarker of Airway Remodelling Modulation in Omalizumab-Treated Severe Asthma Patients. Immunol. Lett. 162 (1), 2-10. doi:10.1016/j.imlet.2014.08.010

Minai-Fleminger, Y., Gangwar, R. S., Migalovich-Sheikhet, H., Seaf, M., Leibovici, V., Hollander, N., et al. (2014). The CD48 Receptor Mediates Staphylococcus aureus Human and Murine Eosinophil Activation. Clin. Exp. Allergy 44 (11), 1335-1346. doi:10.1111/cea.12422

Moore, W. C., Hastie, A. T., Li, X., Li, H., Busse, W. W., Jarjour, N. N., et al. (2014). Sputum Neutrophil Counts Are Associated with More Severe Asthma Phenotypes Using Cluster Analysis. J. Allergy Clin. Immunol. 133 (6), 1557-e5. doi:10.1016/j.jaci.2013.10.011

Morrow Brown, H. (1958). Treatment of Chronic Asthma with Prednisolone Significance of Eosinophils in the Sputum. Lancet 272 (7059), 1245-1247. doi:10.1016/S0140-6736(58)91385-0

Munitz, A., Bachelet, I., Eliashar, R., Khodoun, M., Finkelman, F. D., Rothenberg, M. E., et al. (2006a). CD48 Is an Allergen and IL-3-Induced Activation Molecule on Eosinophils. J. Immunol. 177 (1), 77-83. doi:10.4049/ jimmunol.177.1.77

Munitz, A., Bachelet, I., and Levi-Schaffer, F. (2006b). Reversal of Airway Inflammation and Remodeling in Asthma by a Bispecific Antibody 
Fragment Linking CCR3 to CD300a. J. Allergy Clin. Immunol. 118 (5), 1082-1089. doi:10.1016/j.jaci.2006.07.041

Nair, P., Gaga, M., Zervas, E., Alagha, K., Hargreave, F. E., O’Byrne, P. M., et al. (2012). Safety and Efficacy of a CXCR2 Antagonist in Patients with Severe Asthma and Sputum Neutrophils: a Randomized, Placebo-Controlled Clinical Trial. Clin. Exp. Allergy 42 (7), 1097-1103. doi:10.1111/j.1365-2222.2012.04014.x

Narendra, D., Blixt, J., and Hanania, N. A. (2019). Immunological Biomarkers in Severe Asthma. Semin. Immunol. 46, 101332. doi:10.1016/ j.smim.2019.101332

Neerincx, A. H., Vijverberg, S. J. H., Bos, L. D. J., Brinkman, P., van der Schee, M. P., de Vries, R., et al. (2017). Breathomics from Exhaled Volatile Organic Compounds in Pediatric Asthma. Pediatr. Pulmonol 52 (12), 1616-1627. doi:10.1002/ppul.23785

Noda, S., Suárez-Fariñas, M., Ungar, B., Kim, S. J., de Guzman Strong, C., Xu, H., et al. (2015). The Asian Atopic Dermatitis Phenotype Combines Features of Atopic Dermatitis and Psoriasis with Increased TH17 Polarization. J. Allergy Clin. Immunol. 136 (5), 1254-1264. doi:10.1016/j.jaci.2015.08.015

Nomura, T., Wu, J., Kabashima, K., and Guttman-Yassky, E. (2020). Endophenotypic Variations of Atopic Dermatitis by Age, Race, and Ethnicity. J. Allergy Clin. Immunol. Pract. 8 (6), 1840-1852. doi:10.1016/ j.jaip.2020.02.022

Ortega, H. G., Liu, M. C., Pavord, I. D., Brusselle, G. G., FitzGerald, J. M., Chetta, A., et al. (2014). Mepolizumab Treatment in Patients with Severe Eosinophilic Asthma. N. Engl. J. Med. 371 (13), 1198-1207. doi:10.1056/ NEJMoa 1403290

Planagumà, A., Kazani, S., Marigowda, G., Haworth, O., Mariani, T. J., Israel, E., et al. (2008). Airway Lipoxin A4 Generation and Lipoxin A4 Receptor Expression Are Decreased in Severe Asthma. Am. J. Respir. Crit. Care Med. 178 (6), 574-582. doi:10.1164/rccm.200801-061OC

Price, D., Price, D., Bosnic-Anticevich, S., Briggs, A., Chrystyn, H., Rand, C., et al. (2013). Inhaler Competence in Asthma: Common Errors, Barriers to Use and Recommended Solutions. Respir. Med. 107 (1), 37-46. doi:10.1016/j.rmed.2012.09.017

Price, D. B., Rigazio, A., Campbell, J. D., Bleecker, E. R., Corrigan, C. J., Thomas, M., et al. (2015). Blood Eosinophil Count and Prospective Annual Asthma Disease burden: a UK Cohort Study. Lancet Respir. Med. 3 (11), 849-858. doi:10.1016/S2213-2600(15)00367-7

Ray, A., and Kolls, J. K. (2017). Neutrophilic Inflammation in Asthma and Association with Disease Severity. Trends Immunol. 38 (12), 942-954. doi:10.1016/j.it.2017.07.003

Ricciardolo, F. L. M., Folino, A., Carriero, V., and Bullone, M. (2018). "Role of Blood Neutrophilia as a Biomarker in Asthma," in Monitoring Airway Disease (Sheffield, England: European Respiratory Society), PA1094. doi:10.1183/ 13993003.congress-2018.PA1094

Ricklefs, I., Barkas, I., Duvall, M. G., Cernadas, M., Grossman, N. L., Israel, E., et al. (2017). ALX Receptor Ligands Define a Biochemical Endotype for Severe Asthma. JCI Insight 2 (14). doi:10.1172/jci.insight.93534

Roth, M., and Stolz, D. (2019). Biomarkers and Personalised Medicine for Asthma. Eur. Respir. J. 53 (1), 1802094. doi:10.1183/13993003.02094-2018

Schleich, F., Demarche, S., and Louis, R. (2016). Biomarkers in the Management of Difficult Asthma. Curr. Top. Med. Chem. 16 (14), 1561-1573. doi:10.2174/ 1568026616666151015093406

Schleich, F. N., Zanella, D., Stefanuto, P. H., Bessonov, K., Smolinska, A., Dallinga, J. W., et al. (2019). Exhaled Volatile Organic Compounds Are Able to Discriminate between Neutrophilic and Eosinophilic Asthma. Am. J. Respir. Crit. Care Med. 200 (4), 444-453. doi:10.1164/rccm.201811-2210OC

Serhan, C. N. (2014). Pro-resolving Lipid Mediators Are Leads for Resolution Physiology. Nature 510 (7503), 92-101. doi:10.1038/nature13479

Seys, S. F., Scheers, H., Van den Brande, P., Marijsse, G., Dilissen, E., Van Den Bergh, A., et al. (2017). Cluster Analysis of Sputum Cytokine-High Profiles Reveals Diversity in T(h)2-high Asthma Patients. Respir. Res. 18 (1), 39. doi:10.1186/s12931-017-0524-y

Silvestri, M., Bontempelli, M., Giacomelli, M., Malerba, M., Rossi, G. A., Di Stefano, A., et al. (2006). High Serum Levels of Tumour Necrosis Factor-Alpha and Interleukin-8 in Severe Asthma: Markers of Systemic Inflammation? Clin. Exp. Allergy 36 (11), 1373-1381. doi:10.1111/j.1365-2222.2006.02502.x

Simpson, J. L., Scott, R., Boyle, M. J., and Gibson, P. G. (2006). Inflammatory Subtypes in Asthma: Assessment and Identification Using Induced Sputum. Respirology 11 (1), 54-61. doi:10.1111/j.1440-1843.2006.00784.x
Simpson, J. L., Grissell, T. V., Douwes, J., Scott, R. J., Boyle, M. J., and Gibson, P. G. (2007). Innate Immune Activation in Neutrophilic Asthma and Bronchiectasis. Thorax 62 (3), 211-218. doi:10.1136/thx.2006.061358

Sivakoti, K., Chaya, S. K., Jayaraj, B. S., Lokesh, K. S., Veerapaneni, V. V., Madhunapantula, S., et al. (2018). "Evaluation of Inflammatory Markers MMP-2 and TIMP-1 in Asthma," in Monitoring Airway Disease. (Sheffield, England: European Respiratory Society), PA5044. doi:10.1183/ 13993003.congress-2018.PA5044

Smith, G. M., Biggs, J., Norris, B., Anderson-Stewart, P., and Ward, R. (1997). Detection of a Soluble Form of the Leukocyte Surface Antigen CD48 in Plasma and its Elevation in Patients with Lymphoid Leukemias and Arthritis. J. Clin. Immunol. 17 (6), 502-509. doi:10.1023/a:1027327912204

Tan, Y., Qiao, Y., Chen, Z., Liu, J., Guo, Y., Tran, T., et al. (2020). FGF2, an Immunomodulatory Factor in Asthma and Chronic Obstructive Pulmonary Disease (COPD). Front Cel Dev Biol 8, 223. doi:10.3389/ fcell.2020.00223

Tay, A. S. L., Li, C., Nandi, T., Chng, K. R., Andiappan, A. K., Mettu, V. S., et al. (2021). Atopic Dermatitis Microbiomes Stratify into Ecologic Dermotypes Enabling Microbial Virulence and Disease Severity. J. Allergy Clin. Immunol. 147, 1329-1340. doi:10.1016/j.jaci.2020.09.031

Thijs, J. L., Fiechter, R., Giovannone, B., de Bruin-Weller, M. S., Knol, E. F., Bruijnzeel-Koomen, C. A. F. M., et al. (2019). Biomarkers Detected in Dried Blood Spots from Atopic Dermatitis Patients Strongly Correlate with Disease Severity. Allergy 74 (11), 2240-2243. doi:10.1111/all.13839

Thijs, J., Krastev, T., Weidinger, S., Buckens, C. F., de Bruin-Weller, M., BruijnzeelKoomen, C., et al. (2015). Biomarkers for Atopic Dermatitis: a Systematic Review and Meta-Analysis. Curr. Opin. Allergy Clin. Immunol. 15 (5), 453-460. doi:10.1097/ACI.0000000000000198

Thijs, J. L., van Seggelen, W., Bruijnzeel-Koomen, C., de Bruin-Weller, M., and Hijnen, D. (2015). New Developments in Biomarkers for Atopic Dermatitis. J. Clin. Med. 4 (3), 479-487. doi:10.3390/jcm4030479

Ungar, B., Garcet, S., Gonzalez, J., Dhingra, N., Correa da Rosa, J., Shemer, A., et al. (2017). An Integrated Model of Atopic Dermatitis Biomarkers Highlights the Systemic Nature of the Disease. J. Invest. Dermatol. 137 (3), 603-613. doi:10.1016/j.jid.2016.09.037

Walsh, C. J., Zaihra, T., Benedetti, A., Fugère, C., Olivenstein, R., Lemière, C., et al. (2016). Exacerbation Risk in Severe Asthma Is Stratified by Inflammatory Phenotype Using Longitudinal Measures of Sputum Eosinophils. Clin. Exp. Allergy 46 (10), 1291-1302. doi:10.1111/cea.12762

Weidinger, S., and Novak, N. (2016). Atopic Dermatitis. Lancet 387 (10023), 1109-1122. doi:10.1016/S0140-6736(15)00149-X

Wenzel, S., Ford, L., Pearlman, D., Spector, S., Sher, L., Skobieranda, F., et al. (2013). Dupilumab in Persistent Asthma with Elevated Eosinophil Levels. N. Engl. J. Med. 368 (26), 2455-2466. doi:10.1056/ NEJMoa 1304048

Westerhof, G. A., Korevaar, D. A., Amelink, M., de Nijs, S. B., de Groot, J. C., Wang, J., et al. (2015). Biomarkers to Identify Sputum Eosinophilia in Different Adult Asthma Phenotypes. Eur. Respir. J. 46 (3), 688-696. doi:10.1183/ 09031936.00012415

Wood, L. G., Baines, K. J., Fu, J., Scott, H. A., and Gibson, P. G. (2012). The Neutrophilic Inflammatory Phenotype Is Associated with Systemic Inflammation in Asthma. Chest 142 (1), 86-93. doi:10.1378/chest.11-1838

Conflict of Interest: The authors declare that the research was conducted in the absence of any commercial or financial relationships that could be construed as a potential conflict of interest.

Publisher's Note: All claims expressed in this article are solely those of the authors and do not necessarily represent those of their affiliated organizations, or those of the publisher, the editors and the reviewers. Any product that may be evaluated in this article, or claim that may be made by its manufacturer, is not guaranteed or endorsed by the publisher.

Copyright (c) 2021 Puzzovio and Levi-Schaffer. This is an open-access article distributed under the terms of the Creative Commons Attribution License (CC $B Y)$. The use, distribution or reproduction in other forums is permitted, provided the original author(s) and the copyright owner(s) are credited and that the original publication in this journal is cited, in accordance with accepted academic practice. 